\title{
Dermatitis atópica: una enfermedad emergente
}

\author{
E. Escribano Ceruelo \\ Pediatra. CS Barcelona. Servicio Madrileño de Salud, Área 8. Móstoles, Madrid.
}

Rev Pediatr Aten Primaria. 2009; I I Supl I5:s I I-s4

Eva Escribano Ceruelo, eescribano@pap.es

La dermatitis atópica (DA) es la enfermedad dermatológica crónica más frecuente en la infancia y una de las enfermedades pediátricas más prevalentes ${ }^{1}$. No cabe duda de que en las últimas tres décadas su incidencia ha ido en aumento, en la misma línea que lo han hecho las enfermedades autoinmunes y alérgicas, la llamada epidemia no infecciosa del siglo $X X I^{2}$.

Es una enfermedad compleja en la que la genética y el ambiente están muy involucrados. Permaneciendo invariable la predisposición hereditaria, los cambios en el ambiente, la contaminación y los hábitos de higiene parecen tener un papel muy relevante 3 . Esto es especialmente notable en los países industrializados y más concretamente en las áreas urbanas de los mismos. Los niños nacidos en un país pobre presentan un aumento de atopia cuando emigran a un país rico como el nuestro, sin embargo no será posi- ble predecir cuál será el curso natural de la enfermedad y muy probablemente este no podrá ser modificado.

En España existen pocos estudios de prevalencia, si bien se estima que puede situarse alrededor del $15 \%$ de la población infanti| ${ }^{3,4}$.

Existen aún grandes controversias sobre los diferentes factores etiopatogénicos. Muchos de los factores externos que se consideraban muy relevantes en la fisiopatología de la enfermedad como la lactancia materna, la flora intestinal y el papel de los probióticos y otros alergenos alimentarios han pasado a un lugar menos importante, e incluso se alerta sobre los riesgos de algunos de ellos, para ocupar un lugar más destacado la genética y las anomalías intrínsecas de la piel.

El diagnóstico es exclusivamente clínico y se realiza bien por parte de los pediatras de Atención Primaria, se habla 
incluso de un posible sobrediagnóstico. Sin embargo, es probable que continúe tratándose de forma incompleta; las consultas sobrecargadas, la necesidad de un tiempo razonable para dar una explicación adecuada sobre el curso crónico de la enfermedad, la necesidad de transmitir y perseverar en la importancia del autocuidado, la necesidad de comprender que precisarán cuidados continuados de la piel, la fobia de los pacientes y sus familiares a los tratamientos con corticoides tópicos ${ }^{6} \mathrm{y}$ más recientemente la preocupación por los efectos secundarios de los inmunomoduladores $^{7}$ han contribuido a que los pacientes presenten un auténtico recelo a los diferentes tratamientos y a que estén infratratados. Además, a esto hay que añadir que no se explica bien la correcta forma de administración de las cremas, especialmente en los brotes, por lo que entre unos y otros no se puede conseguir una adecuada adherencia al tratamiento. Además, si la enfermedad tiene un curso grave el tratamiento será complejo y muchas veces frustrante, y tendrán que intervenir otros profesionales como dermatólogos y alergólogos a lo largo de su evolución.

En los casos en los que la enfermedad cursa de forma moderada a grave, esta condición suele repercutir muy negati- vamente sobre la calidad de vida de los enfermos y sus familias ${ }^{8}$, ya que no solo comprometerá al aspecto físico de los pacientes que padecen una enfermedad, visible como otras enfermedades de la piel, sino que también suele afectar muy negativamente al aspecto emocional de padecer una enfermedad cuyo síntoma clave es el prurito, siendo este especialmente nocturno y asociado a menudo a trastornos del sueño. La estigmatización social y laboral y el coste económico ${ }^{9}$ que conlleva su padecimiento deben ser cuidadosamente evaluados, ya que muchos de los tratamientos desgraciadamente no están adecuadamente financiados.

En los últimos años han surgido importantes novedades farmacéuticas que persiguen encontrar un esquema terapéutico diferente al actual y que abogan por un retraso y acortamiento de las recaídas. Pero al mismo tiempo han aparecido diversas alertas de seguridad de determinados medicamentos, situación esta motivo de preocupación esencial en los niños, ya que la seguridad es fundamental; según la OMS tan solo un $10 \%$ de las reacciones adversas a medicamentos son notificadas. Esta organización, en su empeño de promover la seguridad en el empleo de medicamentos en los niños, ha elaborado un docu- 
mento sobre seguridad de los medicamentos en niños, editará una lista de medicamentos esenciales y protocolos de seguridad, y presionará a las compañías farmacéuticas para que pongan en marcha tratamientos específicos en la población infantil ${ }^{10}$.

Ni qué decir tiene la importancia de otros tratamientos alternativos ampliamente extendidos en el cuidado de estos enfermos. En la mayoría de las ocasiones con una seguridad y eficacia limitadas y que habitualmente promueven el abuso a modo de sustancias y cremas "milagrosas" comercializadas fuera de las vías tradicionales bajo el epígrafe de tratamientos "naturales" de la enfermedad y a los que los pacientes se afanan, producto de su desespera- ción por el curso evolutivo de la enfermedad.

Las asociaciones de pacientes han de ocupar ese espacio de información y asesoramiento, así como ser el estímulo para la investigación de calidad en esta enfermedad. La Asociación de Familiares y Pacientes de Dermatitis Atópica (www.adeaweb.org), en 4 años de vida cuenta ya con alrededor de unos 2.800 socios.

En este suplemento monográfico de la Revista Pediatría de Atención Primaria vamos a abordar los avances en la fisiopatología, epidemiología, genética y terapéutica. Asimismo, incluiremos una revisión sobre evidencias en esta enfermedad. Esperamos que nuestras y vuestras expectativas se vean cumplidas.

\section{Bibliografía}

1. Weston WL, Howe W. Epidemology, clinical manifestations, and diagnosis of atopic dermatitis (eczema). UpToDate v.16.3 [consultado el 18/ 02/2009]. Disponible en www.up todate.com

2. Martínez Cócera C. Causas del aumento de las enfermedades alérgicas. Alergol Inmunol Clin. 2002;17(2):1-29.

3. Martín Fernández-Mayoralas D, Martín Caballero JM, García-Marcos Álvarez L. Prevalencia de la dermatitis atópica en escolares de Cartagena y su relación con el sexo y la conta- minación (Estudio ISSAC). An Pediatr (Barc). 2004;60:555-60.

4. Morales Suárez-Varela M, García-Marcos Álvarez L, González Díaz C, Arnedo Pena A, Domínguez Aurrecoechea B. Prevalencia de dermatitis atópica y factores nutricionales en niños de 6-7 años. Aten Primaria. 2007;39: 355-60.

5. Hudson TJ. Skin barrier function and allergic risk. Nat Genet. 2006;38:399- 400.

6. Charman CR, Morris AD, Williams HC. Topical corticosteroid phobia in patients with atopic eczema. Br J Dermatol. 2000;142:931-6. 
7. U.S. Food and Drug Administration. FDA approves updated labeling with boxed warning and medication guide for two eczema drugs Elidel and Protopic (Press release) January 19, 2006. [consultado el 18/02/2009]. Disponible en www.fda.gov/ bbs/topics/news/2006/new01299.html

8. Zuberbier T, Orlow SJ, Paller AS. Patient perspectives on the management of atopic dermatitis. J Allergy Clin Immunol. 2006;118:226-32.

9. Ellis CN, Drake LA, Prendergast MM,
Abramovits W, Boguniewicz M, Daniel CR, et al. Cost of atopic dermatitis and eczema in the United states. J Am Acad Dermatol. 2002;46: 361-70.

10. Promoting Safety of Medicines for Children (WHO). Geneva: WHO; 2007. [consultado el 18/02/2009]. Disponible en www.who.int/ medicines/publications/essentialmedicines/Pro motion_safe_med_childrens.pdf 